Discrete Comput Geom 31:405-410 (2004)

DOI: $10.1007 / \mathrm{s} 00454-003-0796-5$

\title{
No Helly Theorem for Stabbing Translates by Lines in $\mathbb{R}^{3}$
}

\author{
Andreas Holmsen ${ }^{1}$ and Jiří Matoušek ${ }^{2}$ \\ ${ }^{1}$ Department of Mathematics, University of Bergen, \\ Johannes Brunsgt. 12, 5008 Bergen, Norway \\ andreash@mi.uib.no \\ ${ }^{2}$ Department of Applied Mathematics \\ and Institute of Theoretical Computer Science (ITI), Charles University, \\ Malostranské nám. 25, 11800 Praha 1, Czech Republic \\ matousek@kam.mff.cuni.cz
}

\begin{abstract}
For each $n>2$ we construct a convex body $K \subset \mathbb{R}^{3}$ and a finite family $\mathcal{F}$ of disjoint translates of $K$ such that any $n-1$ members $\mathcal{F}$ admit a line transversal, but $\mathcal{F}$ has no line transversal.
\end{abstract}

\section{Introduction}

For a wider background of the questions considered in this note we refer to [12] or other surveys, e.g., [3] and [4], on Helly-type results and geometric transversal theory.

Let $\mathcal{F}$ be a family of convex sets in $\mathbb{R}^{d}$. A line that intersects every member of $\mathcal{F}$ is called a line transversal of $\mathcal{F}$. In 1989 Tverberg [11] proved the following: If $\mathcal{F}$ is a family of disjoint translates of a compact convex set in $\mathbb{R}^{2}$ such that any five members of $\mathcal{F}$ admit a line transversal, then $\mathcal{F}$ admits a line transversal.

This statement was first conjectured by Grünbaum [5] in a paper where he proved a special case, namely, when $K$ is a parallelogram. Prior to this, Danzer [1] proved the case where $K$ is a disk. In 1986 Katchalski [7] proved the weaker statement in which the number 5 is replaced by 128; this was the first Helly-type theorem for line transversals for general translates. It should also be noted that Tverberg's transversal theorem is the best possible, meaning if any of the assumptions are dropped the conclusion no longer holds.

What can be said about line transversals of families of disjoint translates in $\mathbb{R}^{3}$ ? A special case of a theorem by Santaló [10] asserts that if $\mathcal{F}$ is a family of disjoint translates of a parallelotope in $\mathbb{R}^{3}$ and any 20 members admit a line transversal, then $\mathcal{F}$ admits a line transversal. A recent result by Holmsen et al. [6] shows that there is a finite Helly number 
for line transversals to families of disjoint unit balls in $\mathbb{R}^{3}$. However, for translates of a general compact convex set in $\mathbb{R}^{3}$ there is no finite Helly number, as the following theorem shows.

Theorem 1. For any integer $n>2$ there exists a compact convex set $K \subset \mathbb{R}^{3}$ and a finite family $\mathcal{F}$ of disjoint translates of $K$ in $\mathbb{R}^{3}$ such that any $n-1$ members of $\mathcal{F}$ admit a line transversal, and yet $\mathcal{F}$ admits no line transversal.

\section{Proof of Theorem 1}

As almost all previous examples with lines in $\mathbb{R}^{3}$ (for example, Theorem 2.9 in [4]), our construction uses the hyperbolic paraboloid $\Sigma \subset \mathbb{R}^{3}$ with equation $z=x y$ (we imagine the $z$-axis being vertical).

For $a \in\{0,1, \ldots, 6\}$ let $h_{a} \subset \mathbb{R}^{3}$ be the plane $\{x=a\}$. Let $l_{a}$ be the line $h_{a} \cap \Sigma$ and let $s_{a}$ be the segment on $l_{a}$ with $y \in[0,1]$. Note that each line transversal of $\left\{s_{0}, s_{1}, \ldots, s_{6}\right\}$ lies on $\Sigma$ and is one of the lines $\left\{y=y_{0}, z=x y_{0}, y_{0} \in[0,1]\right\}$.

We choose a very small $\varepsilon>0$ and we let $\pi_{\alpha}$ be the vertical plane $\{x=\alpha+\varepsilon y\}$, $0<\alpha<1$. The intersection $\Sigma \cap \pi_{\alpha}$ is the graph of a convex function. Further let $t_{j} \subset \Sigma$ be the line $\{y=j / n, z=x y\}, j=1,2, \ldots, n$; see Fig. 1 . For each $i=1,2, \ldots, n$ we define the $(n-1)$-point set $P_{i}=\left\{p_{i j}: j \in\{1,2, \ldots, n\} \backslash\{i\}\right\}$, where $\left\{p_{i j}\right\}=t_{j} \cap \pi_{i / 2 n}$.

We now introduce conditions on the convex body $K$ and the family $\mathcal{F}$ of translates of $K$. First we want $\mathcal{F}$ to contain translates $K_{a}$ for $a \in\{0,1, \ldots, 6\}$ such that $K_{a} \cap \Sigma=s_{a}$ and $K_{a}$ lies above $\Sigma$ for $a$ even and below $\Sigma$ for $a$ odd.

We claim that every line transversal of $\left\{K_{0}, K_{1}, \ldots, K_{6}\right\}$ is also a transversal of $\left\{s_{0}, s_{1}, \ldots, s_{6}\right\}$. To see this, note that any line transversal $t$ of $\left\{K_{a}: a=0,2,4,6\right\}$ has to lie on or above $\Sigma$ at every section with the plane $h_{a}, a=0,2,4,6$, and since $t$ intersects $\Sigma$ at most twice, it stays completely above $\Sigma$ in at least two of the three intervals

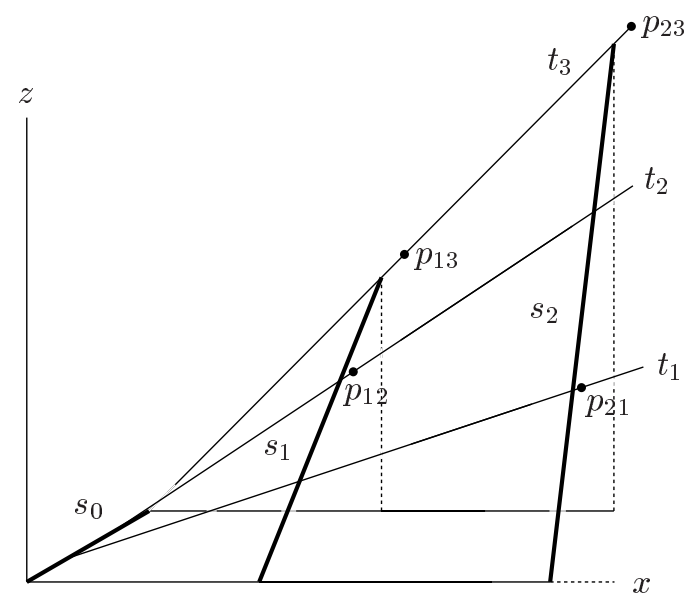

Fig. 1. Some of the objects in the construction (for $n=3$ ). 
$[0,2],[2,4]$, and [4, 6]. Similarly, if $t$ is a transversal of $\left\{K_{a}: a=1,3,5\right\}$, it has to go completely below $\Sigma$ in at least one of the intervals $[1,3]$ and $[3,5]$. Therefore, every line transversal of $\left\{K_{0}, K_{1}, \ldots, K_{6}\right\}$ shares a segment with $\Sigma$, and so it lies completely on $\Sigma$.

Next, we want $\mathcal{F}$ to contain translates $L_{i}$, for $i=1,2, \ldots, n$, such that $L_{i}$ lies above $\Sigma$ and $L_{i} \cap \Sigma=P_{i}$.

Let us assume, for the moment, that there exists a convex body $K$ that satisfies the conditions above such that $\mathcal{F}$ is a family of disjoint translates of $K$. Then each $n-1$ member of $\mathcal{F}$ admits a line transversal, but not the entire family. To see this, note that $t_{i}$ is the unique transversal to $\mathcal{F} \backslash\left\{L_{i}\right\}$, and that $t_{i}$ misses $L_{i}$.

The Construction. Now we give an explicit construction of $K$ that satisfies the conditions above. First we construct a set $X \subset \mathbb{R}^{3}$ consisting of segments $\sigma_{0}, \sigma_{1}, \ldots, \sigma_{6}$ and points $q_{i j}, i=1,2, \ldots, n, j \in\{1,2, \ldots, n\} \backslash\{i\}$, all lying in the narrow vertical slabs $\{0 \leq x \leq \delta\}$, with $\delta>0$ small. Then we define $K=\operatorname{conv}(X)$.

First we let $X_{0}$ be the segment $\sigma_{0}=s_{0}$, lying within the vertical plane $h_{0}=\{x=$ $0\}$. Having constructed $X_{i-1}, i=1,2, \ldots, n$, we obtain $X_{i}$ by appending a suitably translated copy $Q_{i}=\left\{q_{i j}: j \in\{1,2, \ldots, n\} \backslash\{i\}\right\}$ of the set $P_{i}$. This means that we let $m_{i-1}$ be the point of $X_{i-1}$ with the largest $y$-coordinate (e.g., $m_{0}=(0,1,0)$ ), we define $v_{i}$ as the translation vector that moves $m_{i-1}$ to the point of $P_{i}$ with the smallest $y$-coordinate (i.e., $v_{1}=p_{12}-m_{0}$ for $i=1$ and $v_{i}=p_{i 1}-m_{i}$ for $i=2,3, \ldots, n$ ), and we set $Q_{i}=P_{i}-v_{i}$ and $X_{i}=X_{i-1} \cup Q_{i}$. We note that $Q_{1} \cup \cdots \cup Q_{n}$ all lie in the vertical plane $\{y=\varepsilon x+1\}$.

Having constructed $X_{n}$, we similarly append to it translated copies $\sigma_{2}, \sigma_{4}, \sigma_{6}$ of the segments $s_{2}, s_{4}, s_{6}$, respectively, in this order. These lie in the vertical plane $h_{\delta}=\{x=\delta\}$, where $\delta$ is the maximum $x$-coordinate in $X_{n}$; that is, the $x$-coordinate of the last point $q_{n(n-1)} \in Q_{n}$. See Fig. 2(a).

Let $X_{L}$ denote the set constructed so far. For later use, we note that $X_{L}$ is monotone, in the following sense: if $p, q \in X_{L}$ are points, with $y(p)<y(q)(y(p)$ is the $y$-coordinate of $p$ ), then $x(p) \leq x(q)$, with equality only if $p, q$ lie on a common segment $\sigma_{a}$.

Next, we let $X_{U}=\sigma_{1} \cup \sigma_{3} \cup \sigma_{5}$, where $\sigma_{a}$ is a translate of $s_{a}, a=1,3,5$, such that $X_{U}$ lies within the vertical slab $\{x=0,0 \leq y \leq 3\}$, very high above $X_{L}$, and forms the graph of a concave function. Explicitly, $\sigma_{5}=\{x=0, z=5 y+M, 0 \leq y \leq 1\}$, $\sigma_{3}=\{x=0, z=3 y+M+2,1 \leq y \leq 2\}, \sigma_{1}=\{x=0, z=y+M+6,2 \leq y \leq 3\}$, where $M$ is a very large number. Finally, we set $X=X_{L} \cup X_{U}$.

Let $\mathcal{F}_{X}$ be the family of translates of $X$ consisting of:

- $X+v_{i}$, for $i \in\{1,2, \ldots, n\}$, and

- $X+w_{a}$, for $a \in\{1,2, \ldots, 6\}$, where $w_{a}$ is the translation vector with $\sigma_{a}+w_{a}=s_{a}$.

The family $\mathcal{F}$ is obtained by replacing each member of $\mathcal{F}_{X}$ by its convex hull, i.e., a translate of $K=\operatorname{conv}(X)$.

Checking the Properties of $\mathcal{F}$. We now show that the family $\mathcal{F}$ has the desired properties when $\varepsilon>0$ is chosen sufficiently small. For this we first consider the family $\mathcal{F}_{X}$ rather than $\mathcal{F}$. The set $X$ is constructed so that the members of $\mathcal{F}_{X}$ intersect $\Sigma$ exactly the way we want; that is, $\left(X+v_{i}\right) \cap \Sigma=P_{i}$ and $\left(X+w_{a}\right) \cap \Sigma=s_{a}$. 


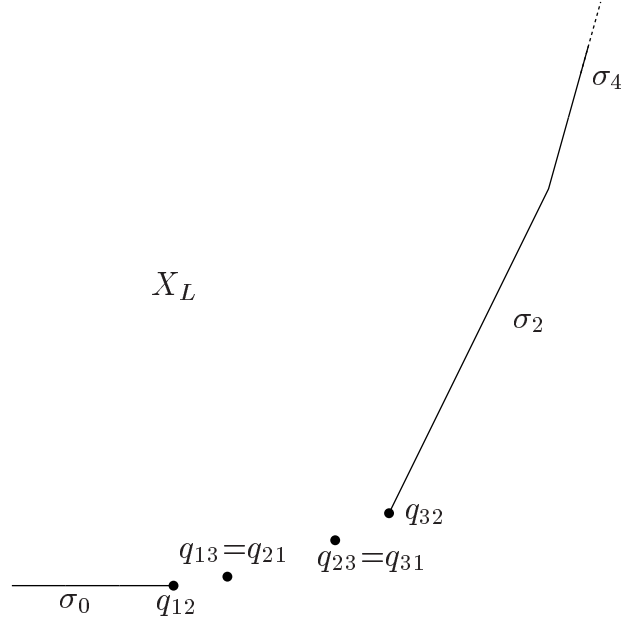

(a)

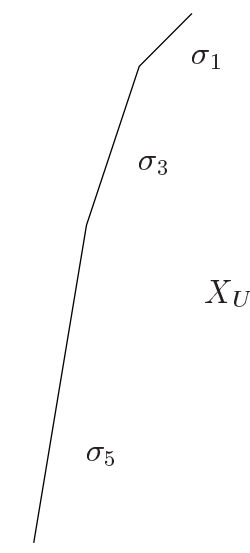

$(0,0, M)$

(b)

Fig. 2. (a) The set $X_{L}$ for $n=3$ (projection to the $y z$ plane); (b) the set $X_{U}$ (shown in a smaller scale than $X_{L}$ ).

To see this, note that the slope (in the $h_{0}$ plane) of the segments $q_{i j} q_{i(j+1)}$ is very close to $i / 2 n$. If follows that each $X+v_{i}$ lies above $\Sigma$ and intersects it only in $P_{i}$. Similarly, we check that for $a=0,2,4,6, X+w_{a}$ lies above $\Sigma$ and intersects it exactly in $s_{a}$, and for $a=1,3,5, X+w_{a}$ lies below $\Sigma$ and intersects it exactly in $s_{a}$.

Clearly, the translates in $\mathcal{F}$ are disjoint, since they lie in disjoint parallel vertical slabs (parallel to the $y z$ plane and of width $\delta$ ). It remains to show that the intersections of the members of $\mathcal{F}_{X}$ with $\Sigma$ are not enlarged by taking convex hulls. We begin with a simple observation:

Observation 2. Let $p, q$ be points lying on or above $\Sigma$, such that $x(p) \leq x(q)$ and $y(p) \leq y(q)$. Then all points of the segment $p q$ lie on or above $\Sigma$. Moreover, an interior point of the segment pq can lie on $\Sigma$ only if $x(p)=x(q)$ and the entire segment pq lies on $\Sigma$.

Proof. To see this, consider the vertical plane $\rho$ passing through $p$ and $q$ (if $x(p)=x(q)$ and $y(p)=y(q)$, then the claim clearly holds, so we may assume that $\rho$ is unique). The intersection $\Sigma \cap \rho$ is the graph of a convex function, so the segment $p q$ lies (nonstrictly) above it. Moreover, that function is strictly convex unless $x(p)=x(q)$ or $y(p)=y(q)$, and in these cases it is a line.

Now we consider a translate $X+v_{i} \in \mathcal{F}_{X}$. First we check that $\operatorname{conv}\left(X_{L}+v_{i}\right) \cap \Sigma=P_{i}$; this is immediate from Observation 2 (if we connect two points in $X_{L}+v_{i}$ by a segment, all interior points of that segment lie strictly above $\Sigma$, and similarly if we connect these "first-generation" points by segments). 
The set $\operatorname{conv}\left(X_{U}+v_{i}\right)$ lies high above $\Sigma$. Any segment connecting a point of $\operatorname{conv}\left(X_{L}+v_{i}\right)$ to $\operatorname{conv}\left(X_{U}+v_{i}\right)$ is almost vertical, and so such segments do not create any new intersections with $\Sigma$. The union of all such segments already equals $\operatorname{conv}\left(X+v_{i}\right)$ (if $A, B \subseteq \mathbb{R}^{3}$ are convex sets, then $\bigcup\{a b: a \in A, b \in B\}$ is convex).

The argument for $\operatorname{conv}\left(X+w_{a}\right) \cap \Sigma=s_{a}, a=0,2,4,6$, is almost identical and we omit it. Finally, $\operatorname{conv}\left(X+w_{a}\right) \cap \Sigma=s_{a}$ for $a=1,3,5$ is easy as well: the situation with $\operatorname{conv}\left(X_{U}+w_{a}\right)$ is clear, as it lives within the vertical plane $h_{a}$, which is intersected by $\Sigma$ in a line, and the contribution of $X_{L}+w_{a}$ is handled as above. This completes the proof of Theorem 1 .

\section{Remarks}

We conclude with some remarks concerning the construction in Section 2.

1. Although our set $K$ is very flat, we note that any $K$ as in Theorem 1 must indeed be three-dimensional. If not, and $\mathcal{F}$ is a finite family of translates of $K$ such that every three members have a line transversal, then either $\mathcal{F}$ lies in a common plane, or all members lie in distinct parallel planes. In both cases there is a finite Helly number: in the first case by Tverberg's transversal theorem, and in the second case by a theorem of Grünbaum (see Theorem 5.6 of [2]).

2. It has already been noted that the members of $\mathcal{F}$ lie in disjoint parallel slabs. Using this observation, labeling the slabs going from left to right, say, gives an ordering of the members of $\mathcal{F}$. By the disjointness of the slabs it follows that any $n-1$ members of $\mathcal{F}$ are met by a line transversal consistently with the ordering. Thus the construction in Section 2 actually proves the following statement (which is stronger than Theorem 1):

Theorem 3. There is no Hadwiger-type theorem for line transversals to families of disjoint translates in $\mathbb{R}^{3}$.

3. Katchalski and Lewis proved the following for families of disjoint translates in the plane: "There exists a constant $k$ such that if every three members of the family admit a line transversal, then there is a line that meets all but at most $k$ members of the family."

Our example can also easily be modified to show that a generalization of this to line transversals in $\mathbb{R}^{3}$ does not exist.

The modification goes as follows. First, instead of the seven segments $s_{0}, s_{1}, \ldots, s_{6}$, we use sufficiently many segments $s_{a}$ so that if any, at most $c$ of them, are removed, a 7-tuple still remains for which the argument in the proof (that any transversal of the corresponding copies $K_{a}$ of $K$ lies on $\Sigma$ ) still works. Second, we choose the parameter $n$ sufficiently large depending on $k$ and $c$, and instead of the simple set system $\{\{1,2, \ldots, n\} \backslash\{i\}: i=1,2, \ldots, n\}$ used for defining the sets $P_{i}$, we use another set system in which every $k$ sets intersect but no $n-c$ do.

4. On the other hand, we do not have an example disproving a fractional Helly theorem of the following type:

There exists a natural number $k$ such that for every $\alpha>0$ there is a $\beta>0$ such that whenever $K$ is a convex body in $\mathbb{R}^{3}$ and $\mathcal{F}=\left\{K_{1}, K_{2}, \ldots, K_{n}\right\}$ is a family 
of disjoint translates of $K$ such that at least $\alpha\left(\begin{array}{l}n \\ k\end{array}\right)$ among the $\left(\begin{array}{l}n \\ k\end{array}\right)$ subfamilies of $\mathcal{F}$ of size $k$ admit a line transversal, then some at least $\beta n$ sets of $\mathcal{F}$ have a line transversal.

This, if true, would be an analogue of the result of Katchalski and Liu [8]; see, e.g., [9] for recent examples of fractional Helly theorems in situations where Helly-type theorems fail.

\section{References}

1. L. Danzer. Über ein Problem aus der kombinatorischen Geometrie. Arch. Math. 8 (1957), 347-351.

2. L. Danzer, B. Grünbaum, and V. Klee. Helly's theorem and its relatives. In Convexity, pp. 101-180. Vol. 7 of Proc. Pure Math. American Mathematical Society, Providence, RI, 1963.

3. J. Eckhoff. Helly, Radon, and Carathéodory type theorems. In Handbook of Convex Geometry, Vol. A, pp. 389-448. North-Holland, Amsterdam, 1993.

4. J. E. Goodman, R. Pollack, and R. Wenger. Geometric transversal theory. In New Trends in Discrete and Computational Geometry, pages 163-198. Springer-Verlag, Berlin, 1993.

5. B. Grünbaum. On common transversals. Arch. Math. 9 (1958), 465-469.

6. A. Holmsen, M. Katchalski, and T. Lewis. A Helly-type theorem for line transversals to disjoint unit balls. Discrete Comput. Geom. 7 (2003), 197-206.

7. M. Katchalski. A conjecture of Grünbaum on common transversals. Math. Scand. 59 (1986), 192-198.

8. M. Katchalski and A. Liu. A problem of geometry in $R^{n}$. Proc. Amer. Math. Soc. 75 (1979), 284-288.

9. J. Matoušek. Bounded VC-dimension implies a fractional Helly theorem. Submitted, 2002.

10. L. Santaló. Un teorema sobre conjuntos de paralelepipedos de arista paralelas. Publ. Inst. Mat. Univ. Nac. Litoral 2 (1940), 49-60.

11. H. Tverberg. Proof of Grünbaum's conjecture on common transversals for translates. Discrete Comput. Geom. 4 (1989), 191-203.

12. R. Wenger. Progress in geometric transversal theory. In Advances in Discrete and Computational Geometry (South Hadley, MA, 1996). Contemp. Math. 223. American Mathematical Society, Providence, RI, 1999.

Received July 17, 2002, and in revised form July 23, 2003. Online publication February 18, 2004. 